\title{
The Detector Safety System for LHC Experiments
}

\author{
Sascha M. Schmeling, Bruce Flockhart, Stefan Lüders, and Giulio Morpurgo
}

\begin{abstract}
The Detector Safety System (DSS), currently being developed at CERN under the auspices of the Joint Controls Project (JCOP), will be responsible for assuring the protection of equipment for the four Large Hadron Collider (LHC) ${ }^{1}$ experiments. Thus, the DSS will require a high degree of both availability and reliability. After evaluation of various possible solutions, a prototype is being built based on a redundant Siemens PLC $^{2}$ front-end, to which the safety-critical part of the DSS task is delegated. This is then supervised by a PVSS $^{3} \operatorname{SCADA}^{4}$ system via an OPC $^{5}$ server. The PLC front-end is capable of running autonomously and of automatically taking predefined protective actions whenever required. The supervisory layer provides the operator with a status display and with limited online reconfiguration capabilities. Configuration of the code running in the PLCs will be completely data driven via the contents of a "configuration database." Thus, the DSS can easily adapt to the different and constantly evolving requirements of the LHC experiments during their construction, commissioning, and exploitation phases.
\end{abstract}

Index Terms-CERN, Detector Control Systems (DCS), detector, experiment, LHC, safety system.

\section{INTRODUCTION}

$\mathbf{T}$ HE Detector Safety System (DSS) project started very late in the development process of the LHC experiments and covered one of the grey areas that still existed, equipment protection. In the beginning of 2001, the four LHC experiments formed a working group to define a project to assure equipment protection for the valuable and sometimes irreplaceable detectors.

The DSS has the task of protecting the experiment's equipment, increase the data-taking efficiency by preventing situations leading to more serious alarms and decreasing downtimes due to system failures, and - in times of low budgets—should not cost too much.

Furthermore, the DSS should be easily integrateable into the control systems of the experiments, be able to connect all subsystem and subdetector safety systems ${ }^{6}$, provide capabilities to easily adapt to the evolving LHC experiments, and be maintainable over the lifetime of the detectors from the first subsystem

Manuscript received June 6, 2003; revised November 5, 2003.

The authors are with the European Laboratory for Particle Physics, CH-1211 Geneva 23, Switzerland (e-mail: Sascha.Schmeling@ cern.ch).

Digital Object Identifier 10.1109/TNS.2004.828631

${ }^{1}$ Large Hadron Collider, accelerator currently being built at CERN.

${ }^{2} \boldsymbol{P}$ rogrammable $\boldsymbol{L}$ ogic $\boldsymbol{C}$ ontroller, standard industry tool for equipment control.

${ }^{3}$ Prozeß-Visualisierungs- und $\boldsymbol{S}$ teuerungs-System made by ETM AG, Austria. www.etm-ag.at

${ }^{4}$ Supervisory $\boldsymbol{C}$ ontrol $\boldsymbol{A}$ nd $\boldsymbol{D}$ ata $\boldsymbol{A}$ cquisition.

${ }^{5} \boldsymbol{O}$ LE for $\boldsymbol{P}$ rocess $\boldsymbol{C}$ ontrol.

${ }^{6}$ It is assumed that all subdetectors of the LHC experiments handle their intrinsic safety themselves. to be tested on-site until the dismantling (a time-span of approximately 20 years).

\section{SCOPE AND GOALS OF A DSS}

During the era of the Large Electron Positron (LEP) ${ }^{7}$ operations, experiment safety was handled by one monolithic safety system. It was decided some years ago to have a CERN-wide system, the CERN Safety System (CSS) for the highest level of safety ${ }^{8}$ [1]. Normal operation of the detectors is performed using the Detector Control Systems (DCS), that handle also low level safety, e.g., the slight overheating of crates. This left a considerably large grey area, especially as the availability and reliability of a PC-based control system does not seem to be sufficient to insure proper equipment protection.

The main goal of the DSS is to detect abnormal and potentially harmful situations, and to minimize the consequent damage to the experimental equipment by taking "protective actions." By implementing this strategy, a reduction of the occurrence of higher level alarms with more serious consequences can be expected, and therefore an increase of the experiment's running time. The DSS should complement and not duplicate existing systems, such as the DCS and the CSS. By working together in a complementary manner, these three systems will insure that situations that may lead to equipment damage, or place people in danger, are well covered. A set of scenarios for DSS interventions can be found in [2].

\section{REQUIREMENTS AND GENERAL ARCHITECTURE}

As a consequence of the aforementioned goals, the following main requirements were defined for the DSS. It has to be

- highly reliable and available, as well as simple and robust;

- provide a cost-effective solution for experimental safety;

operate permanently and independently of the state of DCS and CSS;

- able to take immediate actions to protect the equipment;

- scalable, so that it may grow with the experiment;

- configurable, so that changes in the setup can be accounted for;

- integrated into the DCS, so that existing tools can be reused, the same look and feel is presented to all users, and the monitoring, logging, and presenting are standardized.

\footnotetext{
${ }^{7}$ The Large Electron Positron collider finished physics running in 2000.

${ }^{8}$ According to CERN rules there are three alarm levels (1-3); level 3 is defined as "accident or serious abnormal situation, especially where people's lives are or may be in danger."
} 


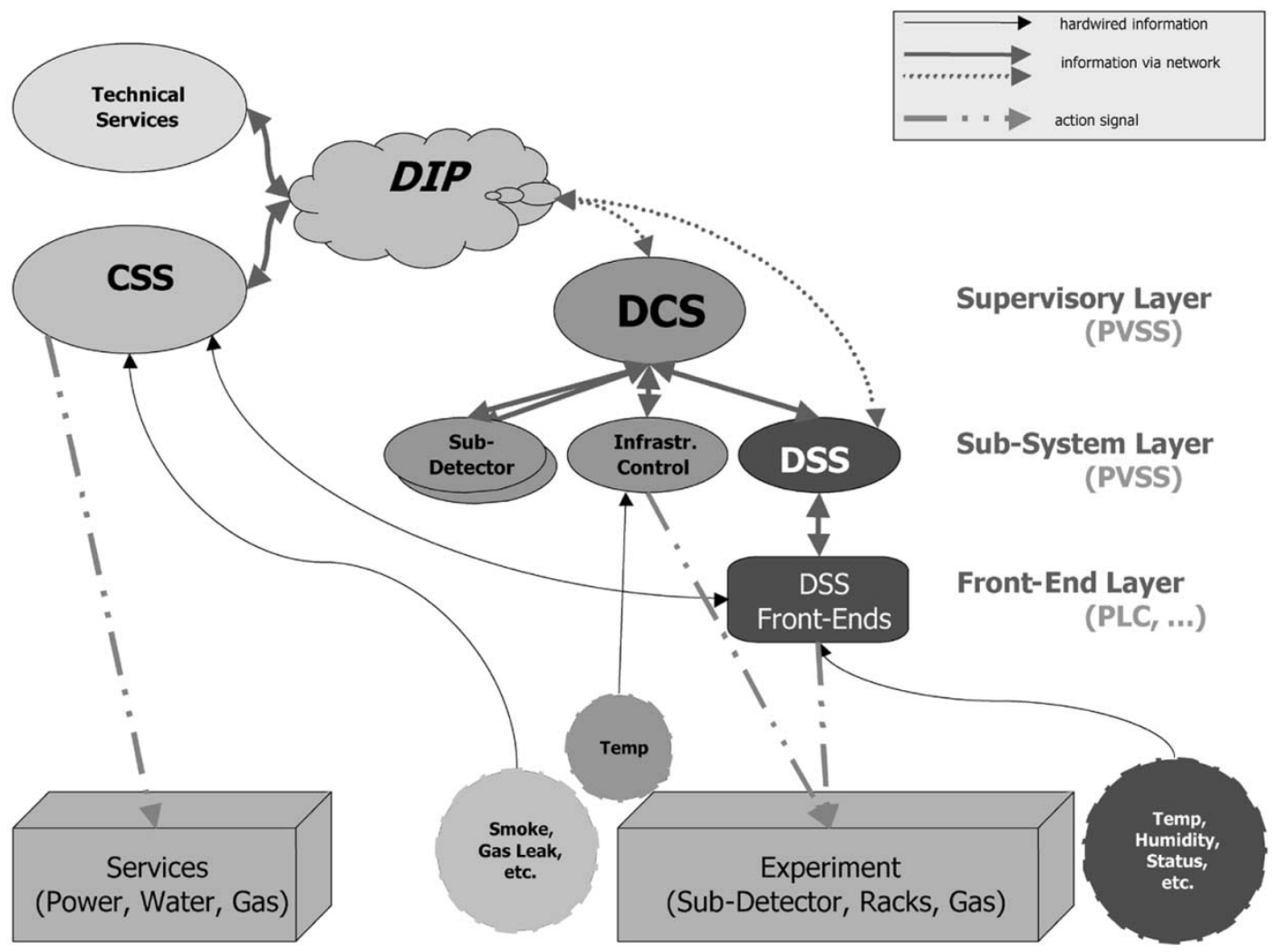

Fig. 1. Scope of the DSS.

Taking all these requirements into account, the DSS was specified by a joint working group between the LHC experiments and the computing support group (IT/CO). Based on the experience from LEP, as well as the very complete survey of technology for high level safety systems at CERN, the following took shape. It will consist of three main entities.

1) The "back-end," which constitutes the User Interface of the DSS and is based on the PVSS SCADA system and the JCOP Framework tools used for configuration, monitoring and logging, user interfaces, and as gateway to external information.

2) The "front-end," to which the safety critical part of the DSS task is delegated. This is composed of several Detector Safety Units (DSU), each responsible for a distinct geographical area, which are built of highly reliable hardware, and which can act upon hardwired information coming from dedicated sensors or from external systems $(\mathrm{CSS}, \ldots)$.

3) The DSS sensors, which collect temperature, humidity and other physical parameters, as well as status information from various external systems.

A complete overview of the DSS inside the detector control system architecture is shown in Fig. 1. It is composed of the following entities:

- the equipment that is acted upon by DCS, DSS, and CSS, subdivided here into two parts, the primary services (e.g., power, water, and primary gas supply) on the lower left-hand side, and the equipment under control of the experiment (e.g., subdetectors, racks, and gas systems);
- the DCS, which is a coherent control system running PVSS on the supervisory and subsystem levels together with their own front-end parts, especially the infrastructure control system, which controls racks, etc., together with its own sensors, e.g., temperature sensors inside the racks;

- the CSS together with its own sensors, taking all required safety actions in case of an alarm of level 3; the Technical Services, here only their respective control systems with interfaces to

a) Data Interchange Protocol (DIP), that provides information exchange between the experiments, the LHC machine, the technical services, and the CSS

b) DSS, consisting of a back-end and a front-end together with its own sensors and autonomous actuators.

\section{IMPLEMENTATION}

The DSS implementation distinguishes between a front-end, and a back-end, which supervises the front-end by a SCADA system. Their intercommunication is performed through a dedicated OPC server/gateway. The general layout for a typical experiment is shown in Fig. 2.

\section{A. Front-End Architecture}

The front-end must be capable of running autonomously and of automatically taking predefined protective actions whenever required. Its design must be as simple and robust as possible, but at the same time highly reliable and available. 


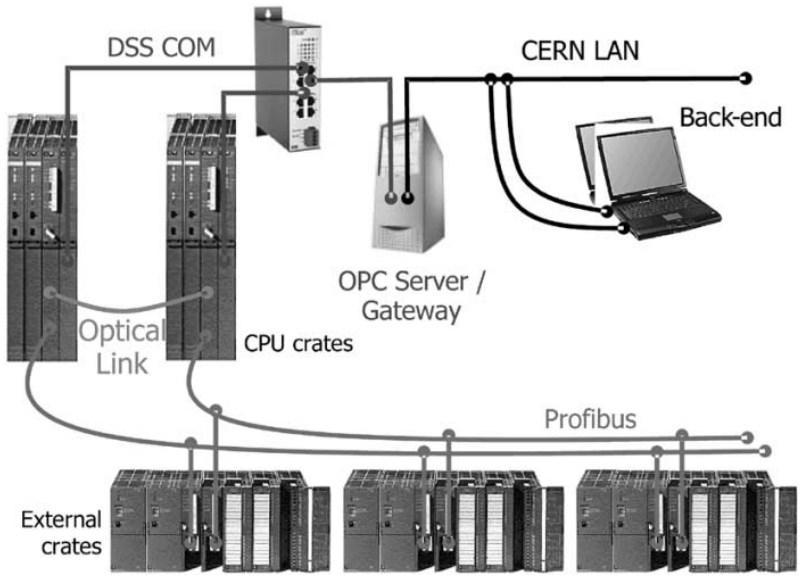

Fig. 2. General DSS Hardware Layout.

The standard used for such applications in industry is "programmable logic controllers" (PLCs). The DSS implementation follows this approach, using a redundant PLC system from Siemens, ${ }^{9}$ which is certified for safety integrity level (SIL) 2 [3] applications. In such systems, the two CPUs constantly monitor and compare their internal states, and automatically detect abnormalities. In the event of a problem, only the nonfaulty CPU continues to operate. The status of the experiment's equipment is monitored by digital and analog sensors (e.g., PT100 for temperature measurement, humidity sensors, and status signals of subdetectors). Required actions are taken by DSS actuators (in this case, usually by cutting the electrical power). The sensors and actuators are dedicated to the DSS.

All parts of the front-end are compliant to the relevant safety norms.

To allow for easy maintenance and for robustness, it was decided not to have equipment other than sensors and actuators in areas which are inaccessible during LHC operation. This choice also implies that there are no strict requirements for the radiation-hardness or magnetic field tolerance of the PLC part. For the exposed sensors and actuators, a variety of industrial components exists, that are certified for the LHC environment.

1) The PLC: The core of the DSS front-end is the redundant PLC system S7-400 H from Siemens. Each CPU of the redundancy pair scans the input signals of the DSS digital and analog inputs attached to the PLC system. Both CPUs run the same DSS Process Code and determine the state of the outputs (the DSS actuators) according to a set of rules defined by the user-the "Alarm-Action-Matrix." This sequence (scanning, processing, and output control) is repeated periodically and is named the "PLC cycle." The cycle time for a typical experiment is of the order of $500 \mathrm{~ms}$, depending on the complexity of the process code and the number of sensors and actuators.

The front-end software can be split into two parts: process code and data blocks. In principle, there are three stages of processing: readout of the sensor values, combination of these

${ }^{9}$ Siemens AG, Germany; www.ad.siemens.com The DSS uses the CPU 414-4H, CP443-1 ethernet communications processor, and the ET $200 \mathrm{M}$ external crate. values into alarms, and the triggering of different actions due to an alarm. For each stage, several data blocks exist, which contain all relevant information on the sensors (e.g., thresholds, physical boundaries, errors, active/inactive), on the actuators (errors, active/inactive), and on the Alarm-Action-Matrix (e.g., the functional relation between inputs, acknowledges of alarms, resets of actions, delays of actions). This information can be modified online at any time via the back-end, and is the basis of the so-called data-driven approach.

The process code is acting on these data blocks in a highly symmetrical manner. Each data block is sequentially accessed and-based on the configured information - the output value for the next stage is determined. The result is sent to the actuators.

The CPU internal state, the input values and the results of intermediate processing steps of both CPUs are monitored and compared regularly during a PLC cycle, by the redundancy process. Differences result in a shutdown of the faulty CPU, which does not affect the DSS itself as the second CPU continues to perform the DSS monitoring task.

Each of the two redundant CPUs is mounted in a CPU crate together with redundant power supplies and Ethernet communication. To achieve synchronization, Siemens interconnects the two CPUs via four (nonredundant) optical fibers. Common time synchronization of both CPUs is achieved by connecting them to the network time server at CERN via the Network Time Protocol (NTP).

Several external crates make up the interface to the DSS sensors and actuators through I/O modules. Since both, sensors and actuators are widely spread over caverns and the surface of the experiment's site, the external crates act as cable concentrators to minimize cable length.

Each external crate can hold up to eight I/O modules. Up to 32 external crates can be connected to both CPUs via a redundant cable pair using the PROFIbus [4] protocol. Thus, redundancy is achieved down to the level of PROFIbus communication. The I/O modules themselves are not redundant, but sensors or actuators can be connected in a redundant fashion by simply doubling their number. External crates and their modules can be connected during DSS operation.

All external crates are powered by a pair of redundant $24 \mathrm{~V}$ power supplies. Their power is also used to supply the current for the sensors and actuators. A power distribution module supplies all parts of the system, while limiting the current to groups of consumers.

2) The Detector Safety Units: The Detector Safety Unit is an assembly of all the above mentioned crates installed in a standard $52 \mathrm{U}$ high rack. This assembly contains the infrastructure to connect sensors and actuators via patch panels, as well as display LEDs for monitoring purposes, and also allows the connection to the Ethernet. Each DSU is capable of monitoring is own state by the use of internal sensors and a dedicated I/O module.

At least one DSU is located on the surface and another in the experimental cavern, so that the two CPUs can be separated to minimize the danger of accidental destruction. The location and the number of DSUs is chosen to minimize the cable installation. 
The layout of all DSUs is the same. The lower half of the rack is dedicated to the patch panel assembly. The upper half hosts an uninterruptible power supply, the OPC server, the CPU crate and two external crates at maximum (this limits the number of DSUs to 16). Only two DSUs contain a CPU crate, and only one the OPC server.

3) Patch Panels: As a large number of sensors can be connected to the DSU, adequate space for cabling is essential. Twenty-six units of rack space inside of the DSU for the patch panel was found to be the optimal solution for serving two external crates or fifteen $\mathrm{I} / \mathrm{O}$ modules that can be inserted in one DSU (two external crates times eight slots, minus one slot reserved for a dedicated monitoring module). Each digital module can handle up to 32 channels; each analog module up to eight channels.

The patch panel terminals from $\mathrm{WAGO}^{10}$ allow for an easy connection of different types of digital and analog sensors. Modifications can be made during the running of the DSS and do not harm the operation itself. All digital channels are galvanically isolated from each other and from the I/O modules by the use of opto-couplers.

352 digital sensors and actuators or 120 analog sensors can be connected to one DSU in total. In the case of the digital channels, the limitations are the space constraints inside the rack and the width of the chosen type of WAGO patch panel terminals. Eleven digital I/O modules with up to 32 channels can be handled. The number of analog channels is limited by the number of free slots in the external crates.

All digital sensors and actuators follow the "Positive Safety" rule. The normal condition is signaled by a "high" level (16-24 V), while alarm conditions give "low" levels (0-5 V). In case of a broken wire, the PLC automatically assumes an alarm condition. Short-circuits or broken wires in the analog sensors are detected by abnormal readings.

4) Uninterruptible Power Supplies (UPS): Each DSU is backed-up by an uninterruptible power supply from APC ${ }^{11}$. Additional battery-packs allow the DSU to be independent of the general power network for at least one hour. This is agreed to be sufficient to bridge the latency until the start of a diesel backed-up power network.

\section{B. The OPC Server and Communication}

The communication between front-end and back-end is routed through a dedicated $1 \mathrm{U}$ high rack-mounted $\mathrm{PC}^{12}$ acting as a gateway. In addition to a 3COM network card, this PC possesses a special Siemens Ethernet adapter (CP1613) to cope with the redundancy of the CPUs: this adapter communicates with both CPUs using ISO protocol. The communication is performed on a dedicated DSS network (DSS COM) using common Ethernet switches. The communication with the

\footnotetext{
${ }^{10}$ WAGO Kontakttechnik GmbH, Germany; www.wago.com

${ }^{11}$ American Power Conversion Corp. (APC), USA; www.apc.com. The DSS uses the APC Smart UPS online 1000VA (APC SUOL1000XLI) with one battery packet (APC SUOL48XLBP) and relay I/O module (APC AP-9610).

${ }^{12}$ Supermicro Computer Inc., U.S.A.; www.supermicro.com. The DSS uses the Supermicro SuperServer 5013G-i.
}

back-end uses the standard CERN network (CERN LAN) connected to the $3 \mathrm{COM}$ card.

The data exchange with the back-end follows the OPC standard [5]. All information exchanged between the PLC and the back-end is handled by an OPC server running on the gateway PC.

\section{Back-End Architecture}

The back-end acts as user interface for the operators in the experiment control rooms and will display the information, acquired via the front-end, as well as supplementary information acquired via the network. In addition, it will also show the procedures to be followed, as well as help screens. Furthermore, it will also assist the experts when modifying the behavior of the safety-relevant front-end.

The back-end is implemented using the PVSS SCADA system. Although all safety-critical aspects are concentrated in the front-end part, the back-end configuration functionality also has a role for safety in limiting the possibilities for operator input errors. This is carried out via an expert-system-like approach, that allows for context analysis and testing to be performed before the download of the new configuration to the front-end. This expert system will evaluate the impact of any configuration change using logical tests and graphical representations to illustrate the full chain from sensors to actuators being configured.

The back-end uses the PVSS database to store the current configuration of the system, while it is planned to store all the modification history into an Oracle Database. This Oracle database will also be used to log the history of all the values of the input channels defined in the system, together with all the alarms detected and the protective actions consequently taken. All the user actions will also be logged.

The operator will be alerted whenever a new alarm is detected. He will have to acknowledge the alarms, and to reset them, once the abnormal situation which triggered the alarm has gone. Subsequently, the operator will be able to take the required actions (probably via the DCS) to bring the detector back into operation.

To ease the work and the understanding of the operator, help pages are foreseen for every alarm, as well as detailed synoptic displays representing the geographical layout of the experiments, and the localization of the alarms and the sensors which generated them.

Finally, the back-end will also be capable of issuing warnings, so that the operator can react in advance to developing situations that could eventually evolve into full alarms.

\section{CONCLUSION}

It has been shown that the initial requirements for the DSS for the LHC experiments could be fulfilled with a relatively simple and robust system. The prototype of the front-end part has been built, and the back-end software part is in progress. This system will undergo a review in the very near future and, afterwards, a small series production will start. In total, about 18 DSUs will 
be built and deployed to the experimental areas in the coming two years, starting in midsummer 2003.

\section{ACKNOWLEDGMENT}

The authors especially want to thank the DSS Working Group $^{13}$ [6] for the excellent collaboration on the definition of the requirements. Furthermore, they would like to thank all the people helping with the finalization of the design and the actual construction of the prototype.

${ }^{13}$ A. Augustinus (ALICE), G. Benincasa (ATLAS), H. Burckhart (ATLAS), Ph. Gavillet (LHCb), R. Nunes (ST/AA), St. Philippin (ALICE), J. Pothier (CMS), W. Salter (JCOP), E. Sbrissa (EP/TA3), Chr. Schäfer (CMS), S. Schmeling (LHCb), L. Scibile (ST/MO), and W. Tejessy (LHCb).

\section{REFERENCES}

[1] "Alarms and alarm systems," CERN, Geneva, Switzerland, CERN TIS/GS, IS37 Rev. 2, May 1998.

[2] "A detector safety system for experiments at the LHC-Scope and architecture overview," DSS Working Group, CERN, Geneva, Switzerland, CERN-JCOP-2002-007, Jan. 2002.

[3] "Functional safety of electrical $\backslash /$ Electronic $\backslash /$ Programmable electronic safety-related systems," CEI-IEC, IEC, Geneva, Switzerland, CEI-IEC-61 508, 1998.

[4] PROFIBUS, Karlsruhe, Germany. PROFIBUS Specification [Online]. Available: http://www.profibus.com/

[5] OPC Task Force, Austin, TX, USA. OLE for Process Control [Online]. Available: www.opcfoundation.org/Downloads/Overview

[6] "A detector safety system for the LHC experiments," CERN, DSS Working Group, Geneva, Switzerland, CERN-JCOP-2002-013, Apr. 2002. 